\title{
MACRÓFITAS AQUÁTICAS DA LAGOA DE JIJOCA DE JERICOACOARA, CEARÁ, BRASIL
}

\author{
Lígia Queiroz Matias ${ }^{1}$ \\ Expedito Rômulo Amado \\ Edson Paula Nunes ${ }^{1}$
}

Recebido em: 26/09/2002. Aceito em: 03/06/2003

\begin{abstract}
RESUMO - (Macrófitas aquáticas da lagoa de Jijoca de Jericoacoara, Ceará, Brasil). A lagoa de Jijoca de Jericoacoara situa-se no litoral do Ceará, Nordeste do Brasil (250'10” e 255'32' S - 40²8'32” e 40²4'09' W), sendo bordejada em sua extremidade Norte por dunas e por vegetação de tabuleiro nas demais faces. Realizou-se o levantamento da composição florística e da estrutura da comunidade de macrófitas aquáticas presentes na lagoa. As amostras das plantas foram coletadas e depositadas no herbário EAC. Densidade, freqüência e cobertura das espécies foram amostradas em 10 transectos plotados em áreas com boas condições de conservação de suas margens, evitando-se áreas urbanas e de atividade agropecuária adjuntas à lagoa. Apresenta-se a lista de 45 espécies ocorrentes, suas formas biológicas e dados da estrutura da comunidade. As espécies com maior índice do valor de importância (IVI) foram Eleocharis mutata (L.) Roem. \& Schult., Paspalidium geminatum Stapf, Eleocharis atropurpurea (Retz.) Kunth, Nymphoides indica (L.) Kuntze e Echinodorus tenellus (Mart.) Buchen. A forma de vida predominante foi "plantas enraizadas ao substrato - anfíbias tolerantes à seca". Em relação à profundidade, ocorre aumento da diversidade de espécies de áreas mais profundas para 0-0,5m de espessura da lâmina d'água.
\end{abstract}

Palavras-chave - macrófitas aquáticas, terras úmidas, lagoas costeiras

\begin{abstract}
Aquatic macrophytes of Jijoca of Jericoacoara lagoon, Ceará, Brazil). The Jijoca of Jericoacoara

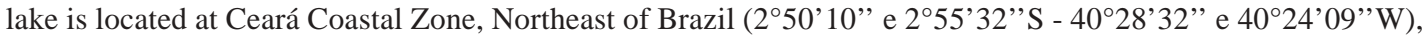
and it has sand dunes on the Northern shore and arboreal semi-deciduous vegetation around the other lakesides. A floristic and communities structure survey of aquatic flora was conducted. Plant samples were collected and included in the EAC Herbarium. The density, frequency, and cover were measurements used in describing communities by intercept transect sampling. Sampling was carried out in Jijoca of Jericoacoara lake at 10 localities, except areas with human impact, such as urban and agricultural areas. A list of 45 species, their life forms and vegetation structure are presented. The higher importance value index (IVI) species were Eleocharis mutata (L.) Roem. \& Schult., Paspalidium geminatum Stapf, Eleocharis atropurpurea (Retz.) Kunth, Nymphoides indica (L.) Kuntze, and Echinodorus tenellus (Mart.) Buchen. The most common life-form was "emergent bottom-rooted plants with amphibious habit". In relation to depth, species diversity increased from the deeper areas and peaked at $0-0,5 \mathrm{~m}$ above water level.
\end{abstract}

Key words - aquatic macrophytes, wetland, coastal lakes

\footnotetext{
Departamento de Biologia, Centro de Ciências, Universidade Federal do Ceará, CEP 60451-970, Fortaleza, CE, Brasil

Bolsista CNPq. UFC, Departamento de Biologia
} 


\section{Introdução}

O litoral cearense está incluído no "Domínio dos depósitos sedimentares cenozóicos" constituído por sedimentos tércioquaternários da Formação Barreiras, sobrepostos a depósitos holocênicos de dunas, praias, mangues e aluviões em superfícies localizadas (Souza 1988), sendo considerado ecossistema de grande fragilidade no contexto do macrozoneamento do litoral brasileiro (MMA 1996).

Algumas lagoas formaram-se nesta região devido à ação conjugada dos processos de avanço de dunas e às variações da linha da costa. Geralmente estas lagoas situam-se em áreas de tabuleiros, sendo bordejadas em sua extremidade por dunas, mantendo contato com o mar através de pequenos cursos d'água, ou por percolação. Em sua maioria são de regime perene e possuem formas meandrantes, por terem sido, anteriormente, um curso fluvial. De forma geral, pode-se afirmar que o litoral do Ceará possui elevado potencial hidrológico, quando comparado com as áreas do semi-árido do Estado. Há riqueza de ambientes aquáticos e anfíbios, devido à perenidade prevalescente da drenagem superficial e a intrusão das águas marinhas nos estuários e lagunas. No entanto, a crescente ocupação do litoral cearense influi para que haja maior preocupação quanto ao uso adequado dos recursos hídricos e a conservação dos ambientes aquáticos (Vicente da Silva 1998).

Segundo Esteves (1998), "nenhuma comunidade límnica foi tão negligenciada no âmbito das pesquisas limnológicas, quanto à formada pelas macrófitas aquáticas". Weaner \& Clements (1938) foram os primeiros a definirem estas comunidades como um conjunto de espécies herbáceas que se desenvolvem em água, em solos cobertos por água ou saturados por esta. Observando o Programa Internacional de Biologia Westlake (1969 apud Esteves 1998), macrófitas aquáticas é a denominação mais adequada para caracterizar vegetais que habitam desde brejos até ambientes verdadeiramente aquáticos, sendo uma denominação genérica, independente de aspectos taxonômicos. Irgang \& Gastal Jr. (1996) adotaram conceito mais amplo do que Cook $(1974 ; 1996)$ ao se referirem às espécies de plantas aquáticas, descrevendo as macrófitas aquáticas como "vegetais visíveis a olho nu, cujas partes fotossintetizantes ativas estão permanentemente ou por diversos meses, ou todos os anos, total ou parcialmente submersas em água doce ou salobra, ou ainda, flutuantes na mesma".

A lagoa de Jijoca de Jericoacoara foi recentemente decretada Área de Proteção Ambiental (Decreto Estadual 25.975/2000) considerando a necessidade de conscientização da população regional sobre a preservação da área e devido à sua riqueza florística, hídrica e paisagística. Outro aspecto relevante consiste na consolidação de ações para o seu desenvolvimento sustentável perante a exploração de seus recursos biológicos naturais, principalmente devido ao turismo. O levantamento da vegetação presente na lagoa realizou-se com o objetivo de observar a diversidade e as formas biológicas das macrófitas aquáticas, assim como a estrutura desta comunidade e a distribuição da flora em relação à profundidade. A partir das informações obtidas, propor sugestões para o manejo adequado deste recurso hídrico.

\section{Material e métodos}

O trabalho foi realizado na Área de Proteção Ambiental da Lagoa de Jijoca de Jericoacoara, localizada no litoral oeste do

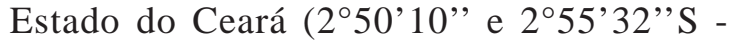
$40^{\circ} 28^{\prime} 32^{\prime \prime}$ e $40^{\circ} 24^{\prime} 0^{\prime \prime} \mathrm{W}$ ), abrangendo área de 3.995,61ha (Fig. 1).

O clima da região é do tipo quente e úmido (Aw‘sg. Köppen 1931), com chuvas de verão ocorrendo de janeiro a julho, e com temperaturas médias oscilando entre $35^{\circ} \mathrm{C}$ e $25^{\circ} \mathrm{C}$, apresentando estação seca com média de duração de 5 a 6 meses, freqüentemente 


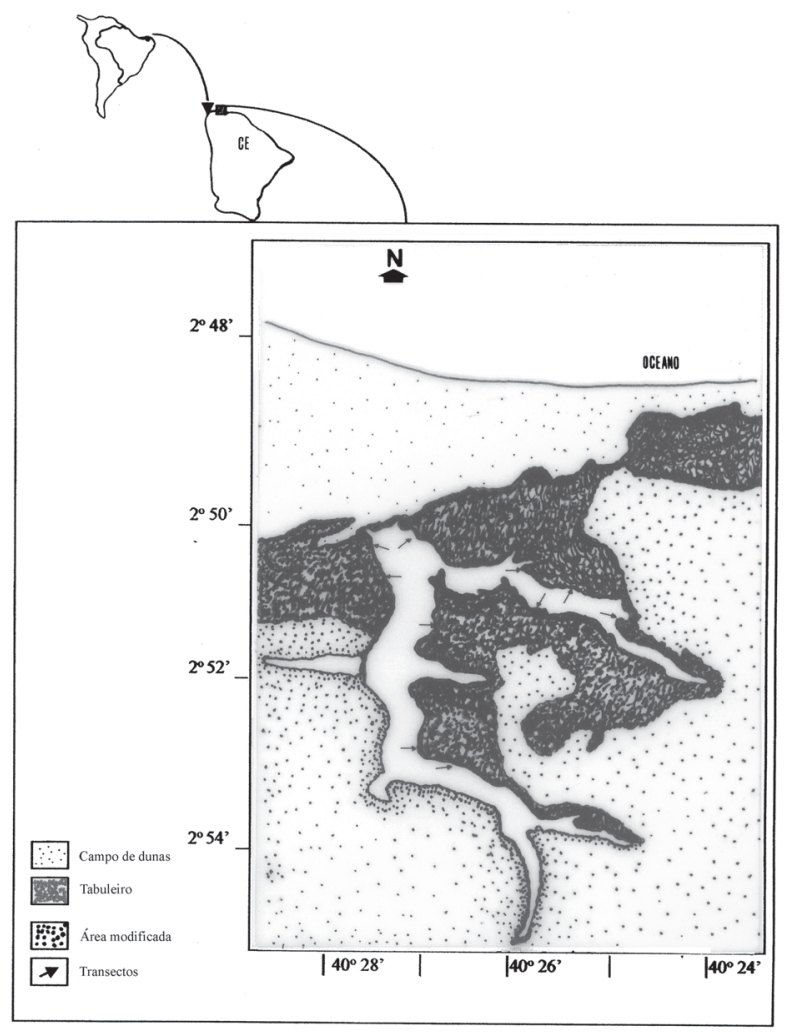

Figura 1. Localização da Área de Proteção Ambiental da Lagoa de Jijoca de Jericoacoara, Ceará, Brasil. (Modificado do Serviço Geográfico do Exército Brasileiro. Folha SA.24.Y.B.IV. 1972. Escala 1:100.000).

interrompida por chuvas ocasionais em setembro e outubro. Os dados referentes ao clima da região foram obtidos durante o ano de 2000, na Estação Meteorológica de Jijoca de Jericoacoara, fornecidos pela Fundação Cearense de Meteorologia e Recursos Hídricos (FUNCEME), de modo a caracterizar o período de estiagem da região e conseqüente regressão da lâmina d'água da lagoa.

$\mathrm{O}$ índice xerotérmico de Gaussen varia entre 100 e 150 (Georgen 1985). A região possui predomínio de sedimentos areno-quartzozos, com vegetação circundante composta por matas de tabuleiro e por vegetação psamófila reptante (Matias \& Nunes 2001).

As coletas foram realizadas nos período de maior precipitação (março a junho) e de seca (agosto a outubro e janeiro) de 2000. A amostragem das comunidades foi definida tendo-se como base o conceito de macrófito aquático (Irgang \& Gastal 1996, mod. Cook et al. 1974), sendo determinadas as plantas submersas ou flutuantes, permanentemente ou não, presentes em toda a lagoa. A metodologia de coleta segue as orientações de Haynes (1984).

O material coletado foi depositado no Herbário EAC (Herbário Prisco Bezerra, Departamento de Biologia, Centro de Ciências, Universidade Federal do Ceará). A identificação foi realizada utilizando-se bibliografia especializada (Caspary 1878; Hoehne 1948; Kral \& Smith 1980; 1982; Fromm-Trinta 1985; 1989; Wiersema 1987; Davidse et al. 1994; Haynes \& Holm-Nielsen 1994; Cook 1996; Luceño, Alves \& Mendes 1997; Rocha 1998). A lista está organizada por ordem alfabética de 
famílias, gêneros e espécies segundo sistema de Judd et al. (1999). A terminologia nomenclatural e abreviações dos nomes dos autores foram feitas segundo INPI (2001). As formas biológicas foram analisadas segundo Irgang \& Gastal Jr. (1996).

Amostrou-se a comunidade por transecção contínua (Brower \& Zar 1984), determinando a composição das espécies desde a borda até a profundidade de $3 \mathrm{~m}$, visto que, após esta profundidade, a ocorrência de macrófitas aquáticas torna-se rara. Foram realizados 10 transectos com auxílio de uma trena e bóia, cada um possuindo $50 \mathrm{~m}$ e disposto em posição perpendicular à margem. Os transectos foram demarcados a partir da interface da lâmina d'água com o solo exposto à época de maior pluviosidade (março) e início das amostragens. As áreas foram definidas tendo-se como parâmetro o estado de conservação de suas margens, evitando-se áreas urbanas ou de intensa atividade agropecuária adjuntas à lagoa (Fig. 1). Obteve-se a identificação das espécies que foram interceptadas pela linha, sendo os indivíduos contabilizados para análise de densidade e frequiência em intervalos de $5 \mathrm{~m}$, de modo que cada transecto possuiu 10 intervalos. Para medida de cobertura, estimouse o "comprimento interceptado" por cada indivíduo. Este comprimento é a porção do transecto interceptado pelas plantas ou pela projeção perpendicular de sua folhagem (Canfield 1941). Em estudos de comunidades herbáceas, observa-se que a estimativa da densidade e cobertura pode ser de difícil interpretação devido a problemas de distinção entre as plantas individualmente. Para superar esta dificuldade, em áreas densamente povoadas e com a presença de espécies clonais, considerou-se como indivíduo cada touceira, indiferente da ocorrência de subunidades geneticamente homólogas, conforme proposta por Pielou (1974).

\section{Resultados e discussão}

O levantamento florístico evidenciou 18 famílias, 33 gêneros e 45 espécies (Tab. 1), número representativo tendo-se como parâmetro os trabalhos de Pott et al. (1989) e de Cervi et al. (1983), porém inferiores aos encontrados por Irgang et al. (1984) e Henriques et al. (1988). A família mais representativa devido ao maior número de espécies foi Cyperaceae, seguida por Fabaceae, Poaceae, Nymphaeaceae e Malvaceae.

Das formas de vida propostas por Irgang \& Gastal Jr (1996), encontrou-se apenas plantas enraizadas ao substrato, sendo predominante as formas anfíbias tolerantes à seca sobre as formas com folhas flutuantes e com partes vegetativas inteiramente submersas (Tab. 1). Entre as plantas com folhas flutuantes encontram-se Nymphoides indica, Nymphaea lasiophylla e Nymphaea rudgeana.

As espécies $N$. indica, Eleocharis mutata e Paspalum vaginatum são comuns nas lagoas costeiras do sudeste brasileiro, as quais também ocorrem na Lagoa de Jijoca de Jericoacoara, sendo reconhecidas como espécies generalistas (Henriques et al. 1988). O mesmo pode-se dizer da espécie Echinodorus tenellus, freqüente nos sistemas aquáticos da América do Sul (Haynes \& Holm-Nielsen 1994).

Ipomoea asarifolia, espécie presente geralmente em regiões dunares (Matias \& Nunes 2001), foi encontrada na forma anfíbia, desenvolvendo raízes adventícias em partes vegetativas submersas. Esta espécie pode contaminar áreas alagáveis, interferindo na dinâmica da comunidade (Burks \& Austin 2000). Porém, na lagoa estudada, ocorreu apenas em áreas de pouca profundidade e sujeitas à seca.

No estudo quantitativo, foram amostradas 22 das 45 espécies presentes na lagoa (Tab. 3), observando-se que as espécies mais frequientes foram Eleocharis sp., E. mutata e Paspalidium geninatum. Estas mesmas espécies apresentam 
Tabela 1. Macrófitas aquáticas da lagoa de Jijoca de Jericoacoara, Ceará. Formas de Vida de Plantas Enraizadas: AT = anfíbias tolerantes à seca, $\mathrm{FF}=$ com folhas flutuantes, $\mathrm{IS}=\mathrm{com}$ partes vegetativas inteiramente submersas.

Família

Gênero/Espécie

Nome vernacular

Forma de vida

Registro EAC

ALISMATACEAE
Echinodorus tenellus (Mart.) Buchen.

BURMANNIACEAE

Burmannia capitata Mart.

lírio d'água

AT

24693

CHARACEAE

Chara sp.

CONVOLVULACEAE

Ipomoea asaralifolia Poir.

CYPERACEAE

Bulbostylis cf. hirtella (Thunb.) Svenson

Cyperus agregatus (Willd.) Endl.

Cyperus amabilis Vahl

Cyperus ligularis $\mathrm{L}$.

Eleocharis aff. geniculata (L.) Roem. \& Schult.

Eleocharis atropurpurea (Retz.) Kunth

Eleocharis barrosi Svenson

Eleocharis elata Boeck.

Eleocharis geniculata (L.) Roem. \& Schult.

Eleocharis mutata (L.) Roem. \& Schult.

Eleocharis sellowiana Kunth

Eleocharis sp. 1

Fimbristylis cymosa $\mathrm{R}$. Br.

Fuirena umbellata Rottb.

Kyllinga vaginata Lam.

alga
salsa-da-praia
capim-barba-de-bode
junco
tiririca
junquinho
junquinho
junquinho
junco-de-cangalha
junquinho
junco-três-quinas
junquinho
junquinho
tiririca
capim

Pycreus polystachyos (Rottb.) P. Beauv.

Rhynchospora cyperoides Mart.

Rhynchospora riparia Boeck

Scleria hirtella $\mathrm{Sw}$.

FABACEAE

Indigofera hirsuta $\mathrm{L}$.

Neptunia plena (L.) Benth

Stylosanthes angustifolia Vog.

Zornia latifolia $\mathrm{Sm}$.

GENTIANACEAE

Schultesia guyanensis Malme

LENTIBULARIACEAE

Utricularia adpressa Salzm. ex. A. St. Hil. \& Girard

Utricularia trichophylla Spruce ex. Oliver

tiririca

tiririca

AT

30244

AT

4868

AT

29510

AT

33121

AT

31.123

AT

29335

AT

29346

AT

29336

AT

29552

AT

31095

AT

29338

AT

29550

IS

AT

31120

IS

31096

AT

31044

AT

29334

31118

AT

29343

AT

29553

AT

29557

AT

29545

AT

31091

anileira

AT

29511

AT

29512

vassourinha

AT

29529

AT

29526

mata-zombando

AT

29514

AT

30245

IS

29549

LOGANIACEAE

Spigelia anthelmia $\mathrm{L}$.

lombrigueira

AT

29498

MALVACEAE

Pavonia cancellata Cav.

Sida anomala A. St. Hil.

malva-rasteira

AT

29499

MENYANTHACEAE

Nymphoides indica (L.) Kuntze

AT

29516

NYMPHAEACEAE

Nymphaea lasiophylla Mart. \& Zucc.

lírio-d'água

FF

29523

ninféia

FF

29546

Nymphaea rudgeana G.F.W. Mey.

nínféia

FF

30216 
Tabela 1 (continuação)

\begin{tabular}{|c|c|c|c|}
\hline $\begin{array}{l}\text { Família } \\
\text { Gênero/Espécie }\end{array}$ & Nome vernacular & Forma de vida & Registro EAC \\
\hline \multicolumn{4}{|l|}{ ONAGRACEAE } \\
\hline Ludwigia octovalvis (Jacq.) Raven & cruz-de-Malta & $\mathrm{AT}$ & 29539 \\
\hline \multicolumn{4}{|l|}{ POACEAE } \\
\hline Paspalidium geminatum Stapf & capim-d'água & AT & 29554 \\
\hline Paspalum vaginatum $\mathrm{Sw}$. & capim & AT & 29519 \\
\hline Panicum sp. & capim & AT & 29551 \\
\hline Paspalum sp. & capim & $\mathrm{AT}$ & 29517 \\
\hline \multicolumn{4}{|l|}{ RUBIACEAE } \\
\hline Borreria verticillata (L.) G. Mey. & vassourinha & AT & 29520 \\
\hline \multicolumn{4}{|l|}{ SCROPHULARIACEAE } \\
\hline Scoparia dulcis L. & vassourinha & $\mathrm{AT}$ & 29501 \\
\hline \multicolumn{4}{|l|}{ STERCULIACEAE } \\
\hline Walteria indica $\mathrm{L}$. & malva-branca & AT & 29503 \\
\hline \multicolumn{4}{|l|}{ XYRIDACEAE } \\
\hline Xyris laxifolia Mart. & botão-d'ouro & $\mathrm{AT}$ & 29556 \\
\hline
\end{tabular}

maior índice de densidade, acompanhadas por Utricularia trichophylla e Echinodorus tenellus. As espécies com maior índice do valor de importância (IVI) foram E. mutata e $P$. geminatum, sendo as espécies que predominam na paisagem da lagoa, ocorrendo em áreas marginais ou em grupos densos emergentes ao centro.

Existem razões para se esperar que a família Cyperaceae seja a de maior riqueza de espécies e, estas, com os maiores IVI na comunidade. Estas espécies possuem sistema subterrâneo complexo formado por rizomas e tubérculos, sendo que algumas dispõem de estolhos subterrâneos, permitindo eficiente propagação vegetativa (Goetghebeur 1998) e, conseqüentemente, representam espécies competitivamente dominantes.

Ocorre um zoneamento na vegetação conforme a espessura da lâmina de d'água, observando maior número de espécies até $0,5 \mathrm{~m}$ de profundidade (Tab. 2). Nesta faixa, que constitui a área de interface entre o ambiente terrestre e o aquático, ocorre maior diversidade de espécies e de formas biológicas, percebendose agrupamentos típicos de vegetação aquática, conforme descrito por Sculthorpe (1967). Pompêu \& Moschini-Carlos (1995), MoschiniCarlos et al. (1993) e Menezes (1984) evidenciaram que a massa de macrófitas aquáticas submersas é baixa quando comparada com a de macrófitas aquáticas emersas em ambientes lênticos do interior de São Paulo. Para a lagoa de Jijoca de Jericoacoara, torna-se provável que o mesmo padrão ocorra, concordando com o modelo de Wetzel (1981). Apenas macroalgas como Chara sp. e espermatófitas como $E$. tenellus, Eleocharis sp.1, E. mutata, N. indica, $P$. geminatum e $U$. trichophylla encontram-se em profundidades maiores, formando agrupamentos homogêneos e característicos destas regiões da lagoa. As espécies de espermatófitas permanecem em estado vegetativo durante o período em que se encontram submersas, exceto $P$. geminatum, que desenvolve colmos flutuantes que permite a emersão dos ramos floríferos, facilmente visíveis em diferentes localidades.

Igualmente como observado por Pott et al. (1989) em lagoas do Pantanal brasileiro, à medida que o nível da lagoa baixa, a vegetação marginal acompanha o recuo 
Tabela 2. Ocorrência das espécies de macrófitas aquáticas da lagoa de Jijoca de Jericoacoara, Ceará, por faixa de profundidade $(+=$ presença; $-=$ ausência $)$.

\begin{tabular}{|c|c|c|c|c|c|}
\hline \multirow[b]{2}{*}{ Espécies } & \multicolumn{5}{|c|}{ Profundidade $(\mathrm{m})$} \\
\hline & $\begin{array}{l}n \\
0 \\
0 \\
0\end{array}$ & $\begin{array}{l}0 \\
n \\
n \\
0\end{array}$ & $\frac{n}{1}$ & $\begin{array}{l}0 \\
i \\
1 \\
n \\
-1\end{array}$ & $\begin{array}{l}\stackrel{0}{i} \\
\stackrel{i}{\wedge}\end{array}$ \\
\hline Burmannia capitata & + & - & - & - & - \\
\hline Cyperus aggregatus & + & - & - & - & - \\
\hline Cyperus ligularis & + & - & - & - & - \\
\hline Chara sp. & - & + & + & + & - \\
\hline Echinodorus tenellus & + & + & - & - & - \\
\hline Eleocharis sp. & + & + & + & + & + \\
\hline Eleocharis atropurpurea & + & - & - & - & - \\
\hline Eleocharis aff. geniculata & + & - & - & - & - \\
\hline Eleocharis geniculata & + & - & - & - & - \\
\hline Eleocharis mutata & + & + & + & - & - \\
\hline Ipomoea asaralifolia & + & - & - & - & - \\
\hline Nymphaea cf. lasiophylla & + & - & - & - & - \\
\hline Nymphoides indica & + & + & - & - & - \\
\hline Panicum sp. & + & - & - & - & - \\
\hline Paspalidium geminatum & - & - & + & + & + \\
\hline Paspalum vaginatum & + & - & - & - & - \\
\hline Pycreus polystachyos & + & - & - & - & - \\
\hline Rhynchospora riparia & + & - & - & - & - \\
\hline Scultesia guyanensis & + & - & - & - & - \\
\hline Scoparia dulcis & + & - & - & - & - \\
\hline Utricularia adpressa & + & - & - & - & - \\
\hline Utricularia trichophylla & + & + & + & + & + \\
\hline Walteria indica & + & - & - & - & - \\
\hline Xyris laxifolia & + & - & - & - & - \\
\hline
\end{tabular}

d'água, havendo também uma sucessão de floração e frutificação das populações. Estes processos ocorrem durante o passar da estação seca que, na região de estudo, ocorre de julho a dezembro (Fig. 2). Neste período, as populações de $U$. trichophylla, Utricularia adpressa, Burmania capitata, E. tenellus tornam-se expostas e, juntamente com Xyris laxifolia, Eleocharis geniculata e E. atropurpurea florescem, de modo que estas foram coletadas férteis apenas sob estas condições ambientais. A determinação da

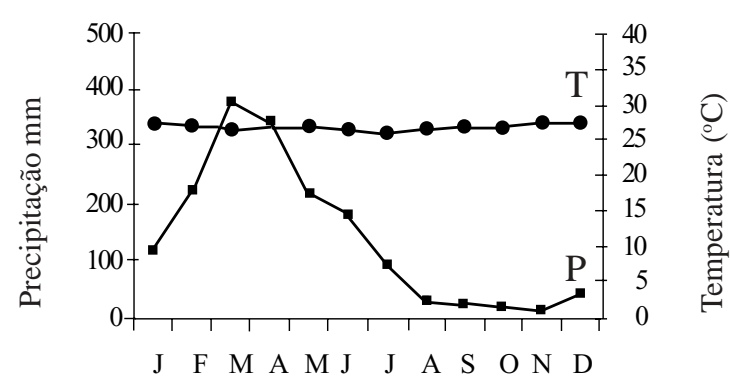

Figura 2. Diagrama climático do Município de Jijoca de Jericoacoara. (•) Temperatura média. ( •) Precipitação média. Fonte: FUNCEME, 2000.

espécie de Eleocharis sp. 1 não foi possível pois, mesmo sob exposição devido ao recuo da lâmina d'água, as populações não floresceram durante o período de amostragem.

De maneira geral, pode-se afirmar que as espécies ocorrem mais comumente em áreas marginais da lagoa, sendo que alguns representantes apresentam período de floração à medida que a lâmina d'água recua, durante a época de seca, que ocorre, geralmente, a partir de julho, como observado na Figura 2. Com a exposição das populações, as plantas tornam-se suscetíveis ao pisoteamento por ocasião da pressão turística, causando impacto às frágeis comunidades vegetais e acarretando a exposição do solo, conforme observado e registrado pelos autores (Matias 2001).

As Cyperaceae, como a família mais diversificada da lagoa e por seus indivíduos possuírem sistema subterrâneo complexo, possui função primordial para a estabilização dos sedimentos, principalmente nas margens, podendo exercer importância estrutural quando ocorrer a recomposição de áreas alteradas. 
Tabela 3. Dados relativos à estrutura da comunidade de macrófitas aquáticas da lagoa de Jijoca de Jericoacoara, Ceará. Número de indivíduos (Ni), Índice de densidade linear (IDL), densidade relativa (DR), frequiência (F), frequiência relativa (FR), índice de cobertura linear (ICL), cobertura relativa (CR), índice do valor de importância (IVI) e em valores percentuais (IVI\%).

\begin{tabular}{|c|c|c|c|c|c|c|c|c|c|}
\hline Espécie & $\mathrm{Ni}$ & IDL & DR & $\mathrm{F}$ & FR & ICL & $\mathrm{CR}$ & IVI & $\operatorname{IVI}(\%)$ \\
\hline Burmannia capitata & 23 & 0,46 & 0,0129 & 0,02 & 0,0142 & 0,0005 & 0,0005 & 0,0276 & 0,920 \\
\hline Cyperus aggregatus & 1 & 0,002 & 0,0006 & 0,02 & 0,0142 & 0,001 & 0,0007 & 0,0155 & 0,517 \\
\hline Cyperus ligularis & 2 & 0,004 & 0,0011 & 0,01 & 0,0071 & 0,004 & 0,0046 & 0,0128 & 0,427 \\
\hline Echinodorus tenellus & 100 & 0,2 & 0,056 & 0,01 & 0,0071 & 0,02 & 0,0229 & 0,086 & 2,867 \\
\hline Eleocharis sp. & 687 & 1,374 & 0,3844 & 0,39 & 0,2766 & 0,137 & 0,1572 & 0,8182 & 27,273 \\
\hline Eleocharis atropurpurea & 78 & 0,156 & 0,0436 & 0,04 & 0,0284 & 0,078 & 0,0892 & 0,1612 & 5,373 \\
\hline Eleocharis geniculata & 3 & 0,006 & 0,0017 & 0,02 & 0,0142 & 0,002 & 0,0017 & 0,0176 & 0,587 \\
\hline Eleocharis elata & 8 & 0,016 & 0,0045 & 0,03 & 0,0213 & 0,005 & 0,0055 & 0,0313 & 1,043 \\
\hline Eleocharis mutata & 345 & 0,69 & 0,1931 & 0,27 & 0,1915 & 0,276 & 0,3158 & 0,7004 & 23,347 \\
\hline Ipomoea asaralifolia & 2 & 0,004 & 0,0011 & 0,01 & 0,0071 & 0,004 & 0,0046 & 0,0128 & 0,427 \\
\hline Nymphaea cf. lasiophylla & 1 & 0,002 & 0,0006 & 0,01 & 0,0071 & 0,001 & 0,0007 & 0,0084 & 0,280 \\
\hline Nymphoides indica & 17 & 0,034 & 0,0095 & 0,12 & 0,0851 & 0,027 & 0,0311 & 0,1257 & 4,190 \\
\hline Panicum sp. & 37 & 0,074 & 0,0207 & 0,03 & 0,0213 & 0,022 & 0,0254 & 0,0674 & 2,247 \\
\hline Paspalidium geminatum & 112 & 0,224 & 0,0627 & 0,19 & 0,1348 & 0,269 & 0,3076 & 0,5051 & 16,837 \\
\hline Paspalum vaginatum & 3 & 0,006 & 0,0017 & 0,01 & 0,0071 & 0,001 & 0,0014 & 0,0102 & 0,340 \\
\hline Pycreus polystachyos & 36 & 0,072 & 0,0201 & 0,02 & 0,0142 & 0,014 & 0,0165 & 0,0508 & 1,693 \\
\hline Rhynchospora riparia & 4 & 0,008 & 0,0022 & 0,02 & 0,0142 & 0,002 & 0,0027 & 0,0191 & 0,637 \\
\hline Scultesia guyanensis & 2 & 0,004 & 0,0011 & 0,01 & 0,0071 & 0,0004 & 0,0005 & 0,0087 & 0,290 \\
\hline Scoparia dulcis & 2 & 0,004 & 0,0011 & 0,01 & 0,0071 & 0,001 & 0,0009 & 0,0091 & 0,303 \\
\hline Utricularia adpressa & 13 & 0,026 & 0,0073 & 0,01 & 0,0071 & 0,001 & 0,0015 & 0,0159 & 0,530 \\
\hline Utricularia trichophylla & 305 & 0,61 & 0,1706 & 0,14 & 0,0990 & 0,006 & 0,007 & 0,2766 & 9,220 \\
\hline Walteria indica & 3 & 0,006 & 0,0017 & 0,01 & 0,0071 & 0,001 & 0,0010 & 0,0098 & 0,327 \\
\hline Xyris laxifolia & 3 & 0,006 & 0,0017 & 0,01 & 0,0071 & 0,001 & 0,001 & 0,0098 & 0,327 \\
\hline Totais $(\Sigma)$ & 1787 & 3,988 & 1 & 1,41 & 1 & 0,8739 & 1 & 3 & 100 \\
\hline
\end{tabular}

\section{Agradecimentos}

À ONG Lagoa Viva e à pousada Feddyssimo, pelo apoio local e hospedagem; ao Prof. Dr. Bruno Irgang, pelas sugestões apresentadas.

\section{Referências bibliográficas}

Burks, K. C. \& Austin, D. F. 2000. Ipomoea asarifolia (Convolvulaceae), another potential exotic pest in the United States. Aquatics 22(2): 16-18.

Brower, J. E. \& Zar, J. H. 1984. Field and laboratory methods for general ecology. Wm.C. Brown Publishers, Iowa.

Canfield, R. 1941. Application of the line intercept method in sampling range vegetation. Journal Forestry 36: 485-494.
Caspary, R. 1878. Nymphaeaceae. In: C.F.P. Martius (ed.). Flora Brasilienseis Leipzig: Monarchii 4(1,2): 120-184.

Cervi, A. C.; Hatschbach, G. \& Guimarães, O. A. 1983. Nota prévia sobre plantas aquáticas (fanerógamas) do Estado do Paraná (Brasil). Boletim do Museu Botânico Municipal 58: 1-17. Cook, C. D. K.; Gut, B. J.; Rix, E. M.; Schneller, J. \& Seitz, M. 1974. Water plants of the world: a manual for the identification of the genera of freshwater macrophytes. The Hargue, W. Junk.

Cook, C. D. K. 1996. Aquatic plant book. SPB Academic Publishing, Amsterdam.

Davidse, G.; Souza, M. \& Chater, A. O. 1994. Flora Mesoamericana: Alismataceae a Cyperaceae 6: 402-500.

Esteves, F. de A. 1998. Fundamentos de Limnologia. $2^{\mathrm{a}}$ ed. Interciência, Rio de Janeiro. 
Fromm-Trinta, E. 1985. Lentibulariaceae do Brasil. Utriculárias aquáticas I. Espécies da região Nordeste. Bradea 5(11): 125-135.

Fromm-Trinta, E. 1989. O gênero Utricularia L. no Brasil III. Espécies da região Nordeste. Bradea 5(17): 188-195.

Georgen, J. 1985. Área de Proteção Ambiental Jericoacoara. PRINTER/CE, UECE/NUGA, SUDEC/DRN, PRMA/DF, PMA/CE, CEDCT/CE, GTZ, Fortaleza.

Goetghebeur, P. 1998. Cyperaceae. Pp. 141-190. In: $\mathrm{K}$. Kubitzki (ed.). The families and genera of vascular plants. Springer, Berlin.

Haynes, R. R. \& Holm-Nielsen, L. B. 1994. The Alismataceae. Flora Neotropica 64: 1-112.

Haynes, R. R. 1984 Techniques for collecting aquatic and marsh plants. Annals of the Missouri Botanical Garden 71: 229-231.

Henriques, R. P. B.; Araújo, D. S. D.; Esteves, F. A. \& Franco, A. C. 1988. Análise preliminar das comunidades de macrófitas aquáticas da Lagoa Cabiúnas, Rio de Janeiro, Brasil. Acta Limnológica Brasileira 2: 783-802.

Hoehne, F. C. 1948. Plantas aquáticas. Instituto de Botânica de São Paulo, São Paulo.

INPI - International Plant Names Index. [on line] Disponível na Internet via www.url: http:// www.ipni.org. Consulta realizada em 15/outubro/2001.

Irgang, B. E. \& Gastal Jr., C. V. de S. 1996. Macrófitas aquáticas da planície costeira do RS. UFRGS, Porto Alegre.

Irgang, B. E.; Pedralli, G. \& Waetcher, J. L. 1984. Macrófitos aquáticos da Estação Ecológica do Taim, Rio Grande do Sul, Brail. Roessléria 6(1): 395-404.

Judd, W. S.; Kellog, E. A. \& Stevens, P. F. 1999. Plant systematics: a phylogenetic approach. Sinauer Associates, Sunderland.

Köppen, W. 1931. Climatologia. Fondo de Cultura Económica, Buenos Aires.

Kral, R. \& Smith, L. B. 1980. Xyridaceae brasiliae I. Bradea 3(9): 57-64.

Kral, R. \& Smith, L. B. 1982. Xyridaceae brasiliae I. Bradea 3(34): 273-289.

Luceño, M.; Alves, M. V. \& Mendes, A. P. 1997. Catálogo florístico y claves de identificación de las ciperáeas de los Estados de Paraíba y Pernambuco (Nordeste del Brasil). Annales del Jardín Botánico de Madrid 57: 67-100.

M. M. A. 1996. Macrodiagnóstico da zona costeira brasileira. Ministério do Meio Ambiente e da Amazônia Legal, Brasília.
Matias, L. Q. 2001. Macrófitas aquáticas da Lagoa de Jijoca de Jericoacora, CE e suas implicaçõs para o manejo e conservação ambiental. Relatório Técnico, Universidade Federal do Ceará, Organização Não-Governamental Lagoa Viva.

Matias, L. Q. \& Nunes, E. P. 2001. Levantamento florístico da Área de Proteção Ambiental de Jericoacoara, Ceará. Acta Botanica Brasilica 15(1): 35-43.

Menezes, C. F. S. 1984. Biomassa e produção primária de três espécies de macrófitas aquáticas da represa do Lobo (Broa), SP. Dissertação de Mestrado. Universidade Federal de São Carlos, São Carlos.

Moschini-Carlos, V. 1991. Aspectos ecológicos da associação vegetal de Scirpus cubensis na Lagoa do infernão-SP. Dissertação de Mestrado. Universidade Federal de São Carlos, São Carlos.

Pielou, E. C. 1974. Population and community ecology. Gordon and Breach Science Publishers, New York.

Pompêo, M. L. M. \& Moschini-Carlos, V. 1995. Zonação e biomassa das macrófitas aquáticas na Lagoa Dourada (Brotas, SP), com ênfase na Utricularia gibba L. Acta Limnologica Brasiliensia 7: 78-86.

Pott, V. J.; Bueno, N. C.; Pereira, R. A. C.; De Salis, S. M. \& Vieira, N. L. 1989. Distribuição de macrófitas aquáticas numa lagoa na fazenda Nhumirim, Nhecolândia, Pantanal, MS. Acta Botanica Brasilica 3(2) Supl.: 153-168.

Rocha, E. A. 1998. Estudo taxonômico da seção Teneus do gênero Rhynchospora Vahl (Cyperaceae) no Brasil. Dissertação de Mestrado. Universidade Federal de Pernambuco, Recife.

Schulthorpe, C. D. 1967. The biology of aquatic plants. Edward Arnold, London.

Souza, M. J. N. 1988. Contribuição ao estudo das unidades morfo-estruturais do estado do Ceará. Revista de Geologia 1: 73-91.

Vicente da Silva, E. 1998. Geoecologia da paisagem do litoral cearense; uma abordagem ao nível de escala regional e tipológica. Tese para concurso de Professor Titular, Departamento de Geografia, Universidade Federal do Ceará, Fortaleza.

Weaner, J. E. \& Clements, F. E. 1983. Plant Ecology. Mc. Graw Hill, New York.

Wetzel, R. G. 1981. Limnologia. Omega, São Paulo. Wiersema, J. H. 1987. A monography of Nymphaeaeceae subgenus Hydrocallis (Nymphaeaceae). I. Four new species from the neotropics. Brittonia 36(3): 213-222. 